\title{
Subadditive concatenation in dissimilarity judgments
}

\author{
PETER H. SCHÖNEMANN \\ Purdue University, West Lafayette, Indiana \\ and University of Bielefeld, Bielefeld, West Germany \\ TIMOTHY DORCEY \\ Purdue University, West Lafayette, Indiana \\ and \\ KIM KIENAPPLE \\ Mount St. Vincent University, Riverdale, New York
}

\begin{abstract}
Empirical results are presented that point toward consistent violations of intradimensional ("segmental") additivity in dissimilarity judgments about rectangles. These results contradict all Minkowski power metrics, and support a recently introduced "metric for bounded response scales" (MBR) that predicts intra- and interdimensional subadditivity. We found strong indications of systematic individual differences. We therefore analyzed the individual dissimilarity judgments for four groups separately, first at the within-subjects level and later at the group-average level. The stability with which our results replicated across the independent groups served as a consistency and robustness check. Since numerous metrics other than the MBR might account for segmental subadditivity, we compared the fit of four versions of the MBR, the three major Minkowski metrics, and two alternative segmentally subadditive metrics. The city-block metric uniformly gave the worst fit. We obtained the best fit for an MBR that reduces the upper bound by about one-fifth of the response scale range. No significant improvement was obtained by permitting the upper bound to vary from subject to subject. Although the orientation question (whether subjects employed a height/width or an area/shape system) could not be resolved conclusively, it appeared that the largest group used height and width, whereas the second-largest group used shape predominantly.
\end{abstract}

Direct dissimilarity ratings on category scales are often used as basic input for multidimensional scaling (MDS). A number of different theories have been proposed to explain such dissimilarity judgments. For example, Restle (1961) assumed that stimuli could be characterized in terms of sets of features (or "aspects") that enable subjects to utilize some set norm to arrive at their responses. Because this theory does not invoke order relations, it is not tied to a geometric representation of the stimuli in a finite-dimensional space. Restle's theory has been favorably received in Europe (e.g., Eisler \& Roskam, 1977; Ekman, 1963; Micko \& Fischer, 1970), and it has been generalized by Tversky (1977) and Tversky and Gati

The revision of this paper was completed while the senior author was on sabbatical leave in Germany, which was made possible by a grant from the Deutsche Forschungsgemeinschaft (DFG). He wishes to thank the DFG, and also Professors K. Müller (Munich), U. Schulz (Bielefeld), and C. Micko (Braunschweig) for sponsoring him. Finally, we thank Professor E. Roskam (Nijmegen) for his prompt and generous response to our request for his MRSCAL program, and Associate Editor M. Teghtsoonian for her comments on a previous draft and her detailed suggestions for improving the style and legibility of this paper.

P. H. Schönemann's mailing address is: Department of Psychological Sciences. Purdue University, West Lafayette, IN 47907.
(1982). For lack of space, this set-theoretic approach cannot be discussed further here.

In contrast, Attneave (1950) proceeded from the basic assumption that dissimilarity judgments take place in a finite-dimensional geometric space that enables subjects to concatenate the perceived line segments $a, b$ (see Figure 1), which the stimulus pairs project onto the axes of an underlying subjective coordinate system. This theory is order-dependent and appears especially plausible for stimuli that can be described in terms of one or more physical dimensions. Attneave used as stimuli geometric forms that systematically varied along two such objective dimensions. In his analysis, he focused his attention on two particular members of the Minkowski family of distance functions, the Euclidean and city-block distance (Householder-Landahl hypothesis, Attneave, 1950, p. 521). This line of reasoning has been expanded by Beals, Krantz, and Tversky (1968), Hyman and Well (1967), Krantz and Tversky (1975), Shepard (1962), and Wender (1969, 1971), among others. The Krantz and Tversky study, in particular, has spawned numerous follow-up studies (Borg \& Leutner, 1983; Noma \& Johnson, 1977; Schönemann, 1977, 1979, 1980, 1982, 1983; Schönemann \& Borg, 1981; Schönemann \& Kienapple, 1984; Takane, 1981; 


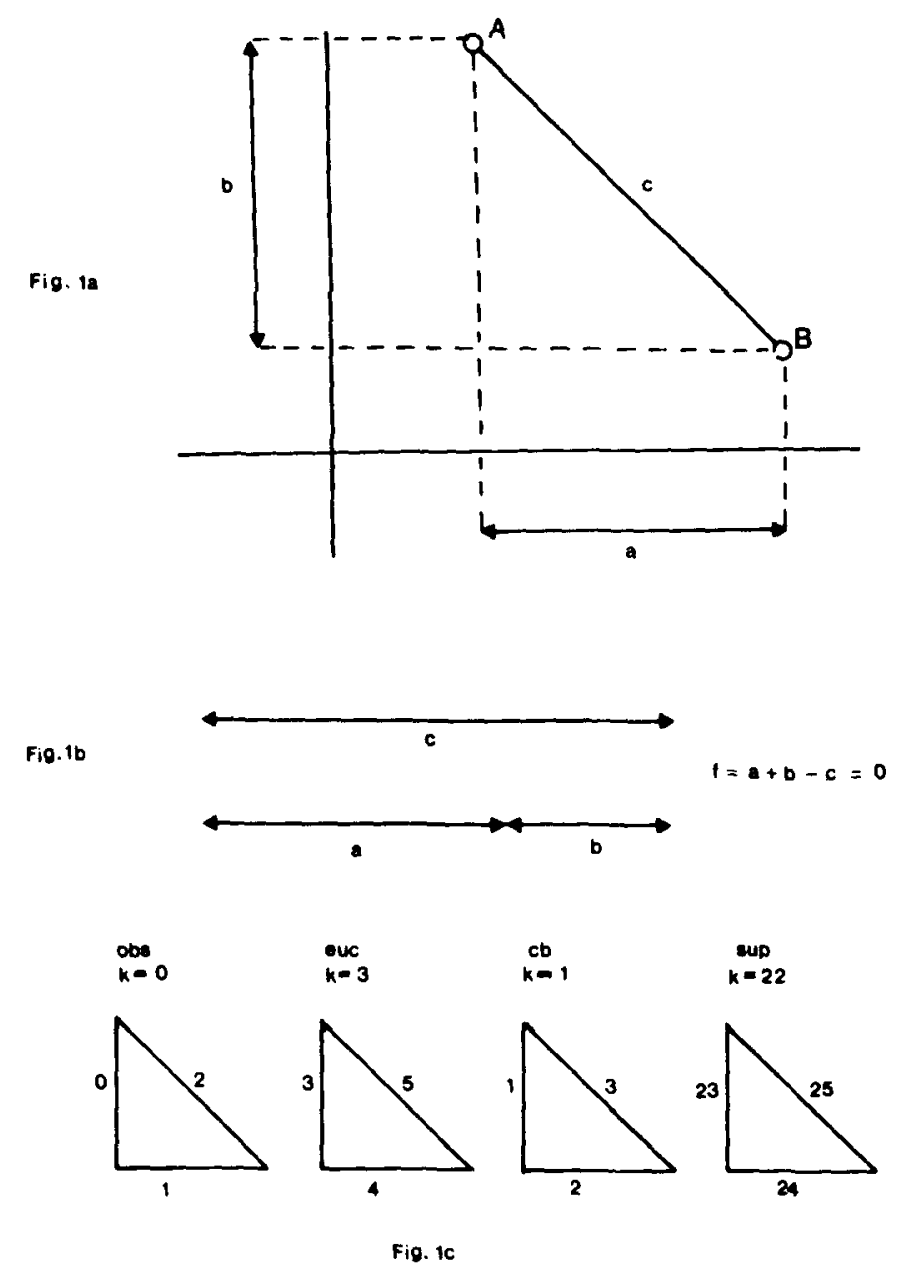

Figure 1. (a) Notation for metrics: the distance $c$ between points $A$ and $B$ is expressed as a function of the projections $a, b$, that is, $c=g(a, b)$. (b) For three collinear points, the function $f=a+b-c$ will be zero if the distance $g(a, b)$ is segmentally additive, otherwise it will be positive. If it is negative, the triangle inequality is violated. (c) Effect of additive constant on Minkowski metrics (see text).

and Wiener-Ehrlich, 1978), including the experimental work to be reported here.

Within this framework, two major problems that arise are: (1) the metric problem-one has to determine the appropriate metric before the stimuli can be represented in an m-dimensional vectorspace; and (2) the orientation problem-one has to establish the orientation of the presumed subjective coordinate system, because most metrics, with the notable exception of the Euclidean metric, are not invariant under rotation of the coordinate system.

Attneave (1950) limited the metric question to the Minkowski family, without, however, giving any psychological explanation for singling out this particular class of metrics. So far as we know, only Wender (1969) has made an attempt to justify their use psychologically in a controlled experiment. He argued that power metrics with larger exponents were more likely to arise under degraded perceptual conditions. For example, when the stimuli are exposed for only a brief moment, it becomes difficult to attend to all dimensions, and only the most salient projections-in the limiting case, only the largest ("supremum" or "sup metric") - enter into the dissimilarity judgments. Conversely, under the most favorable perceptual conditions, all dimensions receive equal attention, so that the city-block metric is more likely to fit. However, the empirical evidence Wender cites in support of these predictions does not strike us as very strong.

The present study was designed to shed further light on the metric problem and the orientation problem. It emulates the Krantz and Tversky (1975) study, which, in turn, can be viewed as an extension of similar work by Sixtl and Wender (1964) and Wender $(1969,1971)$. Krantz and Tversky presented subsets of pairs of 17 rectangles to two groups of subjects and asked them to indicate the perceived dissimilarity on a 20 -point rating scale. The rectangles 
had been constructed so that the design formed a rhombus in an objective log-height and log-width coordinate system (see Figure 2a). ${ }^{1}$

Krantz and Tversky first checked a number of ordinal "decomposability" conditions that are necessary for a very general class of composition rules to apply (see Beals et al., 1968). These conditions were violated in both coordinate systems. Since this class of composition rules included all Minkowski power metrics as special cases, the data were incompatible with all Minkowski metrics. In the expectation "that, unlike the Euclidean model, the INDSCAL program yields a preferred set of psychological dimensions" (Krantz \& Tversky, 1975, p. 29), the authors then used Carroll and Chang's (1970) INDSCAL program for an MDS analysis. The result was a fan-shaped stimulus configuration in the subjective plane similar to that shown in Figure $2 d$.

On the basis of the MDS analysis, Krantz and Tversky concluded (1) that their subjects utilized area and shape, rather than height and width, in arriving at their numerical dissimilarity estimates, and (2) that "the main failure of decomposability ... is the tendency for an interval along one dimension to appear larger the higher or more extreme the level of the orthogonal dimension"' (p. 31). That is, area and shape interacted in the sense that shape differences became more accentuated with increased area. This finding echoes similar results reported by Wender (1971).

However, there now exists converging evidence that this A/S interaction may have been an artifact induced by the stimulus design and the logarithmic pretransformation of the H/W coordinates: (1) The INDSCAL program implements a variant of a subjective metrics model, first proposed by Horan (1969), that squarely rests on the Euclidean metric and hence on decomposability. Thus, Krantz and Tversky seem to confront their readers with a contradiction: in the first part of their paper they reject decomposability of their data on the basis of the ordinal
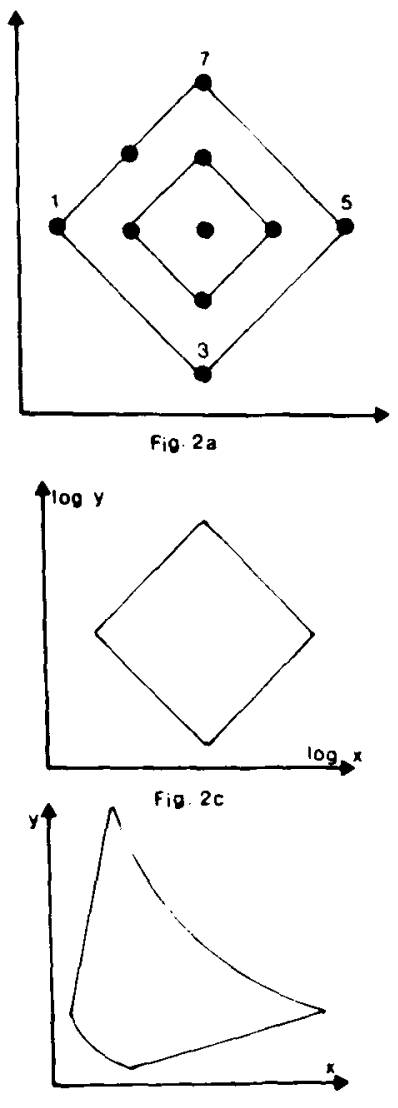

Fig. 2d



Fig. 2b

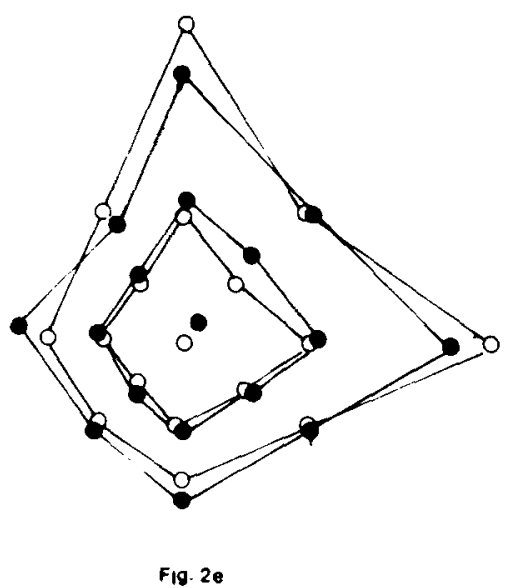

Figure 2. Interaction artifact of the Krantz and Tversky (1975) study on rectangles. (a) Schematic stimulus design of the study in $\log (\mathrm{W}) / \log (\mathrm{H})$ coordinate system. (b) MDS configuration. Stimulus configuration appears rotated by $45^{\circ}$ into a $\log ($ area $) / \log ($ shape) coordinate system, and shape differences become larger with increasing area (apparent area/shape interaction). (c-d) The same apparent interaction effect can be produced by simply reversing the log transformation of the physical design coordinates. (e) Least squares fit of the delogarithmized design (open circles) to MDS configuration $2 \mathrm{~b}$ (closed circles) under choice of a similarity transformation. 
tests, whereas in the second part they argue for an $\mathrm{A} / \mathrm{S}$ interaction on the basis of an MDS analysis that rests on Euclidean distances and hence on decomposability. (2) Although this seeming contradiction can be resolved by applying a nonlinear, instead of an affine, transformation to the objective H/W system to restore decomposability (Schönemann, 1977, 1979, 1980), Krantz and Tversky's claim that the underlying dimensions were $A / S$, rather than $\mathrm{H} / \mathrm{W}$, is more difficult to justify. Horan's model rests on two fairly strong conditions, a common-space condition and a diagonality condition. The orientation of the coordinate system for a fixed number of dimensions is identified only if the diagonality condition holds (Schönemann, 1972; Schulz, 1971, 1975). Since the authors did not test these constraints (for a program and norms permitting such tests, see Schönemann, James, \& Carmer, 1978), their conclusion, based on the INDSCAL analysis, that their subjects used the $\mathrm{A} / \mathrm{S}$ system is open to question. (3) Once the rotational question is reopened, the empirical support for the claimed interaction effect erodes, because Schönemann and Borg (1981) produced a very similar A/S interaction effect from the design alone by simply reversing the $\log$ transformation of the objective $\mathrm{H} / \mathrm{W}$ coordinates and subsequently rotating back into a H/W system (see Figures 2c-2e). This means the Krantz and Tversky data are also consistent with the trivial hypothesis that their subjects simply used height and width veridically, rather than area and shape interactively, as Krantz and Tversky have claimed. (4) At least superficially, this trivial hypothesis received additional support in a recent independent replication by Borg and Leutner (1983). They used two different, completely crossed stimulus designs, one for $\mathrm{H} / \mathrm{W}$ and another for $\mathrm{A} / \mathrm{S}$, on two groups of subjects. The authors reported faster responses for the $\mathrm{H} / \mathrm{W}$ group than for the $\mathrm{A} / \mathrm{S}$ group and found virtually no interactions for the $\mathrm{H} / \mathrm{W}$ group. Borg and Leutner therefore concluded: "There is no need to introduce 'interaction effects' into a dimensional model of rectangle perception if (1) one uses a width/height system, with (2) appropriately rescaled physical units and (3) the city-block metric as a composition rule" (Borg \& Leutner, 1983, p. 264).

While on first blush these results seem to confirm the postmortem analysis of Schönemann and Borg (1981), the Borg and Leutner study also raises some new questions.

Borg and Leutner "expect that Model 1 fits best if $p=1$ [where $p$ is the Minkowski exponent; i.e., they expect the city-block metric to fit best], because there is ample evidence . . . that the city-block metric is the adequate distance function for 'analyzable' stimuli, such as rectangles" (p. 260). If this statement were taken literally, then Borg and Leutner's own data provide ample evidence contradicting it. Table 5 gives the means and average sums of squared residuals (SSQ) left by the three major Minkowski metrics for the four possible collinear comparisons (along height, width, and the two diagonals of the designs) relative to the SSQ left by the city-block metric. In terms of these ratios, the Euclidean metric fits 71 times better than the city-block metric for the height comparisons in the
H/W group, 29 times better for the width comparisons, and 25 times better for the diagonal comparisons. Similar, though perhaps less dramatic, results were found for the two triangular comparisons and the A/S group. Moreover, the residual means for the three possible collinear comparisons are all positively biased, averaging roughly .2 on a $[0,1]$ scale. Since all Minkowski metrics are segmentally additive, the residual means would have to be zero for at least one of the collinear comparisons. Hence, none of the Minkowski metrics can fit the Borg and Leutner data, regardless of whether a $\mathrm{H} / \mathrm{W}$ or an $\mathrm{A} / \mathrm{S}$ system is assumed. In particular, Borg and Leutner's claim that the city-block metric describes their data is not just unsupported but is strongly contradicted by their data.

Thus, it appears that even with such simple and wellcontrolled stimulus material as rectangles, both the metric question and the orientation question are still wide open. In order to make further progress, and to keep the discussion concise, a few definitions will be needed. We shall say data (or metrics and norms) are "subadditive" for three points, A, B, and C, if the sum of the dissimilarities a (between A and B) and $b$ (between B and C) exceeds the dissimilarity $\mathrm{c}$ (between $\mathrm{A}$ and $\mathrm{C}$ ) (see again Figure 1), so that the function

$$
f=a+b-c
$$

is positive. We shall say they are "additive" if this function is zero, and "superadditive" if it is negative. Thus, superadditivity is equivalent to violations of the triangle inequality. With the exception of the city-block metric, which is additive both between and within dimensions, all Minkowski metrics are additive within, but subadditive between, dimensions. For the Borg and Leutner data (Table $5, \mathrm{CB}$ column for residual means), as well as for our own data to be reported here (Tables 2-3), the function $f$ is predominantly positive. This means that dissimilarity judgments about rectangles tend to be subadditive both between and within dimensions, regardless of whether a $\mathrm{H} / \mathrm{W}$ or an $\mathrm{A} / \mathrm{S}$ system is assumed.

When one of Borg and Leutner's reviewers drew attention to the subadditivity in their data, they dismissed it as having only minor importance: "It was not assumed that the data were distances, and with ordinal and even interval data, such violations of triangle equalities can at least be reduced by subtracting a sufficiently large constant from the Ds. ... [T] he low S [stress] values indicate that the distances are in excellent agreement with the admissibly transformed data. Hence, the observed 'violations' . . are, at most, of minor importance, since they can be largely eliminated by admissibly transforming the data into their monotone regression values" (p. 264).

Thus, when Borg and Leutner speak of a "best fit" with the city-block metric, they do not mean a fit to the actually observed data, but rather to monotonically distorted data, in keeping with the presently dominant "nonmetric" philosophy of MDS. However, we should not lose sight of the fact that the intervening monotone transformations are completely ad hoc, psychologically unexplained, and 
a priori unknown. Their sole purpose is to enhance the appearance of a good fit. In invoking monotone transformations, the investigator voluntarily and often quite unnecessarily restricts his range of admissible inferences severely, because, strictly speaking, any claims about the fit would always have to be qualified with a reference to this large class of transformations: instead of speaking of a "best fit with the city-block metric," it would be more appropriate to speak of a "best fit of the city-block metric cum unexplained ad hoc monotone transformation."

One casualty of these ad hoc transformations is the metric problem, especially if it is opened up to encompass all possible metrics, instead of restricting it quite arbitrarily to the Minkowski family. These metrics constitute only a vanishing fraction of all possible metrics. With the possible exception of Wender (1969), few seem to have bothered to explain why this particular distance family should be singled out as especially appropriate for psychology.

It is not difficult to see that the mechanical interpolation of an unknown monotone transformation between data and a spatial model makes it virtually impossible to diagnose the metric: Monotone transformations can always be chosen so as to favor any particular metric. To see this for the Minkowski family, consider Figure 1a. If one denotes the two projections of the stimulus interval onto the coordinate axes a,b, the city-block metric can be written

$$
\mathrm{d}_{\mathrm{c}}=\mathrm{a}+\mathrm{b},
$$

the Euclidean metric

$$
d_{e}=\left(a^{2}+b^{2}\right)^{1 / 2},
$$

and the sup metric

$$
\mathrm{d}_{\mathrm{s}}=\max \{\mathrm{a}, \mathrm{b}\} .
$$

If the observed dissimilarities are $(0,1,2)$, the triangle inequality is violated. By adding 3 to these values, one obtains the triple $(3,4,5)$, which gives an exact fit for the Euclidean metric. However, by adding only 1 , one obtains $(1,2,3)$, and thus a perfect fit for the city-block metric. Finally, by adding a very large constant, for example, 22 , one obtains $(23,24,25)$, which approximates the sup metric. The fit will improve with the magnitude of the additive constant (Figure 1c). In short, since the questions of the appropriate monotone transformation and the appropriate metric are confounded, we cannot answer both simultaneously. Hence, if one seeks an answer to the metric question, one has to look at the actual data.

\section{METRICS FOR BOUNDED RESPONSE SCALES}

There is no obvious reason why the data should be distances of any kind, even if we instruct the subjects to produce distances. Usually, only the triangle inequality is in serious doubt (although some authors have also questioned the symmetry axiom for certain types of dissimilarities). In the older literature, considerable effort was invested in the "additive constant problem": how best to transform superadditive dissimiliarities monotonically so that they would satisfy at least the triangle inequality, and perhaps also some additional constraints. It now appears that this focus may have been misplaced. Most data we have had a chance to analyze were subadditive, not superadditive. The triangle inequality is usually satisfied within sampling error, and the main problem is that intradimensional additivity is violated because dissimilarity data are consistently subadditive.

It was this observation that prompted Attneave to subtract an additive constant from his observed dissimilarities. However, he offered no psychological explanation for the necessity of this subtraction. He believed that "if these quantities are to be considered 'distances' in any meaningful sense, they should be additive along any single continuum" (Attneave, 1959, p. 524). This is true for the Minkowski family, but not for distances in general. The three distance axioms (nonnegativity, symmetry, and triangle inequality) say nothing about intradimensional additivity, nor do they imply it. A well-known example of a segmentally subadditive metric is the chord metric: Consider three points along a great circle on the surface of a sphere. If the distances are measured on this surface (arc metric), they will be additive. If, instead, they are measured as Euclidean distances across the inside of the sphere, they will be segmentally subadditive (chord metric).

Schönemann (1982, 1983) advanced a psychological hypothesis to explain why category ratings of dissimilarities often tend to be subadditive. A new metric for bounded response scales (MBR) was derived from two basic assumptions: (1) Subjects try their best to make some sense out of the ambiguous experimental instruction to produce "dissimilarity ratings on a scale from $x$ to $y$," but they are able to perform only elementary binary operations, such as simple geometric concatenation ("putting end to end") of perceived intradimensional differences. This assumption would seem to favor the simple city-block metric as a process model $(c=a+b)$. However, if the dissimilarities have been obtained as category ratings ("Please tell me the dissimilarity on a scale from 0 to 9"), the instruction imposes an upper bound on the responses a subject is allowed to make ( 9 , in this case). (2) To respect this bound, subjects have to contract the larger perceived projections on the coordinate axes before they can concatenate them so that the result fits the category scale provided by the experimenter. ${ }^{2}$ More generally, such bounds may also be self-imposed by the subjects, or they may be imposed by nature, that is, by certain physiological limitations of the sensory systems.

Therefore, if such data are to be explained in terms of a city-block-like composition rule at all, then this rule must provide for a subadditive concatenation of the perceived coordinate segments. A relatively simple mathematical 
map that meets these desiderata is used in physics to describe the addition of velocities which, according to Einstein's special relativity theory, never exceed the speed of light. When all velocities are divided by this upper bound, the relative velocities vary between 0 and 1 and they add according to the addition rule for hyperbolic tangents:

$$
\tanh (u+v)=\frac{\tanh (u)+\tanh (v)}{1+\tanh (u) \tanh (v)}, \quad 0 \leq u, v \leq 1
$$

Schönemann $(1982,1983)$ has invoked this bounded composition for the analysis of dissimilarity data by applying it to the projections a,b (Figure 1) so as to obtain the "metric for bounded response scales" (MBR), which, in terms of relative distances $(0 \leq d \leq 1)$, can be written

$$
\text { MBR: } d_{m}=(a+b) /(1+a b),
$$

where $a=\tanh (u), b=\tanh (v), 0 \leq a, b \leq 1$, and more generally, in terms of observed distances $d^{*}$,

$$
\operatorname{MBR}(k): d_{m}=\left(a^{*}+b^{*}\right) /\left(1+a^{*} b^{*} / k^{2}\right),
$$

where $a^{*}, b^{*}$ are now the projections onto the coordinate axes in the original metric and $\mathbf{k}$ is the upper bound. By permitting $\mathrm{k}$ to vary across subjects, one obtains a oneparameter family of subject-specific MBRs.

On applying the same reasoning to contiguous-line segments within one dimension, one obtains a subadditive norm (measure of length) within dimensions of the same form (Equation 7). By "'norm," we mean here any nonnegative map into the reals that satisfies the triangle inequality $[n(a)+n(b) \geq n(a \oplus b)$, where $\oplus$ denotes the concatenation] but not necessarily homogeneity [norm(sA) $=|\mathrm{s}|$ norm(a) $]$, although homogeneity is usually required for norms on linear spaces.

Schönemann $(1982,1983)$ showed that this concatenation is symmetric, associative, and invertible over $[0,1) \in \operatorname{Re}$, and that it predicts overestimation for halving, underestimation for doubling, and an upward shift of the midpoint for middling. It was further shown that the MBR (Equations 6 and 7) is indeed a metric, that it is monotone in both arguments, and that it approximates the cityblock metric for small arguments, the sup metric for large arguments (near k), and the Euclidean metric for intermediate arguments. This suggests a reason why Minkowski metrics often appear to give reasonable fits even though they are all unbounded.

To apply the MBR in practice, it is necessary only to first express all distance relative to the upper bound $k$ (which need not be the same for all subjects). In view of Equation 5, an inverse hyperbolic tangent transformation (Fisher's z-transform) of the relative distances will then restore additivity because it leads to the composition rule for the city-block metric $\left[\tanh ^{-1}\left(\mathrm{~d}_{\mathrm{m}}\right)=\mathrm{u}+\mathrm{v}\right]$. The transformed data can therefore be analyzed with any one of the standard MDS programs (e.g., MRSCAL, Roskam, 1972 ) in the city-block mode without invoking any additional unknown monotone transformations. Hence, if the MBR hypothesis (that the subjects utilize a composition similar to that of the MBR) is approximately correct, the fit of the city-block metric should improve after an inverse hyperbolic tangent transformation of the relative dissimilarities. The fit comparison should, of course, not be based on any indirect stress measure, which automatically divides out all monotone transformations, but rather on the differences between actually observed data and reproduced MBR distances.

It has been asked what gain derives from entertaining the hyperbolic tangent transformation (Equations 6 and 7) over simply using a conventional MDS program that also interpolates a monotone transformation to analyze the data. The main difference between the approach advocated here and conventional MDS is that our monotone transformation (Equations 6 and 7) is the explicit consequence of a psychological hypothesis about how subjects arrive at their numerical responses. This hypothesis leads to a specific prediction, namely segmental subadditivity, which can be tested. In contrast, routine employment of a nonmetric MDS program does not permit such a test, because the program will remove the subadditivity with a monotone transformation (the inverse tanh transformation, if the MBR hypothesis holds) before the fit is evaluated in terms of stress. This is the reason, we presume, why few so far have paid attention to the pervasive subadditivity of dissimilarity ratings, even though such data have been analyzed innumerable times with iterative nonmetric MDS programs.

A second, related, difference is that the class of monotone transformations (Equations 6 and 7) is relatively narrow. Equation 7 is a one-parameter family and Equation 6 is parameter-free. In contrast, the class of all possible monotone transformations that conventional MDS programs interpolate is vast. Since the monotone transformations are unspecified a priori, they convey no empirical information about the subject's behavior; they only make the fit look better. The price one pays for invoking this vast class of transformations is loss of falsifiability of potentially interesting psychological hypotheses that might explain how the subjects arrived at their numerical responses. It is apparently not universally realized that any increase in generality exacts a price.

On the other hand, even if our MBR hypothesis were qualitatively correct, the mathematical function used to model the data need not be that of the hyperbolic tangent transformation. There are innumerable other mathematical functions that satisfy the three distance axioms and respect an upper bound k. Many of these will be very close to Equation 7 over the range $[0, \mathrm{k})$. At this point, our choice of Equation 7 is pragmatic and is motivated mainly by the fact that the inverse hyperbolic tangent transformation restores additivity in a convenient way, leading back to the city-block metric. If another subadditive metric were consistently to give a better fit than Equation 7, then 
it should replace the hyperbolic tangent transformation as an MBR. We view the question of which mathematical function best describes subadditive data as a purely empirical question. Since it assumes greater weight as the evidence for subadditivity in dissimilarity ratings accumulates, we will pursue the question in greater detail in the next section, in which a number of different subadditive metrics will be empirically compared with several versions of the MBR.

\section{ALTERNATIVE SUBADDITIVE METRICS}

The fit of four versions of the MBR is compared with the fit of a number of other metrics, including the three major Minkowski metrics and two alternative subadditive metrics that might qualify as plausible models to account for subadditive dissimilarities. In this section, we define these alternative metrics and describe some of their formal properties. The following nine functions $g(a, b)$ and acronyms will be used in our empirical evaluation:

$\mathrm{CB}=\mathrm{a}+\mathrm{b}$ : city-block metric.

SUP $=\max \{a, b\}:$ sup metric.

EUC $=\left(a^{2}+b^{2}\right)^{1 / 2}:$ Euclidean metric.

$\operatorname{MBR}(\mathrm{L})=$ Equation 7, with $\mathrm{k}=\mathrm{L}$, the largest admissible response ( 9 for our data): MBR with fixed bound. To save space, we use "M(L)" instead of " $M B R(L)$ " in the numerical tables, and similarly " $M(\mathrm{sL})$, ," "M(MX)," "M(sMX).,

$\operatorname{MBR}(s L)=$ Equation 7, with $k=s L$ and $s$ a proper fraction (we shall use $s=.8$ ): MBR with reduced fixed bound.

$\operatorname{MBR}(\mathrm{MX})=$ Equation 7 , with $\mathrm{k}=\mathrm{MX}$, the largest dissimilarity rating of a given subject: subject-specific MBR.

$\operatorname{MBR}(\mathrm{sMX})=$ Equation 7, with $\mathrm{k}=\mathrm{MX}$, the largest dissimilarity given by the subject, multiplied by the proper fraction s (.8): subject-specific MBR with reduced bound.

AVER $=(a+b) / 2$ : average.

AVPW $=\left(a^{1 / 2}+b^{1 / 2}\right) / 2$ : average power.

$\operatorname{MBR}(\mathrm{sL})$ is motivated by the observation that, in category rating tasks, subjects typically do not utilize the whole response range if it is large (see, e.g., Figure 3.6 in Parducci, 1982, p. 98), so that the self-imposed "true upper bound" may be slightly lower than that provided by the experimenter. MBR(MX) allows for individual differences. Some subjects may be more conservative than others in utilizing the response scale provided by the experimenter, and thus may employ a self-imposed smaller bound that varies from subject to subject. MBR(sMX), in addition, allows for sampling error in the actually observed maximal dissimilarity. Since $k$ governs the curvature of the MBR function, the fit may be improved by lowering this value slightly to obtain a more reliable upper bound for the subject-specific response scale. AVER has been suggested by Anderson's (1970) repeated find- ing that subjects prefer averaging over summation in simple concatenation tasks (for cogent criticisms of some of Anderson's claims, see Birnbaum, 1982, and Gigerenzer, 1983). AVPW is a concatenation with a built-in concave contraction reminiscent of Stevens's power law (Plateau 1872). To impose the bound, the average was used. AVER and AVPW could of course be generalized to one-parameter families, if desired.

The conventional definitions of CB, SUP, and EUC as power metrics require intradimensional additivity. However, even if this requirement is dropped, all nine functions remain metrics, because they all satisfy the three familiar distance axioms. We shall therefore retain the descriptors SUP and EUC also for the segmentally subadditive versions, and treat the conventional sup and $\mathrm{Eu}$ clidean metrics as special cases. With this proviso, all nine metrics (including EUC) identify the orientation of the coordinate system; that is, their only isometrics are reflections and translations. For the segmentally additive EUC, one has to add the rotation group.

The critical test for segmental additivity, and thus for the whole family of Minkowski metrics in the conventional sense, is provided by $C B=a+b$, since for this metric we have $\mathrm{f}=\mathrm{a}+\mathrm{b}-\mathrm{c}=\mathrm{a}+\mathrm{b}-\mathrm{CB}=0$ for all comparisons. For all other Minkowski metrics, this relation must hold within dimensions.

If viewed as binary operations in $[0,1) \in R e$, these functions $g(a, b)$ can be characterized in terms of a number of formal properties, such as additivity or subadditivity, associativity, monotonicity, and boundedness. We call a concatenation "monotone" if it is monotone in both arguments, a,b, so that the result is never less than $\max \{a, b\}$, and "strictly monotone" if it is larger, for positive a,b. We say a concatenation "respects the upper bound" if the result of $n$-fold concatenation of any element with itself never exceeds an upper bound $\mathrm{k}$. Using this terminology, some of the formal properties of the above nine metrics are summarized in Table 1 .

To see that the average is not monotone, consider $\operatorname{AVER}(\mathrm{a}, \mathrm{b})=,(.9+.1) / 2=.5<.9 \max \{.9, .1\}$. Similarly, AVPW is not monotone (take $a=.81, b=.16$ ). The MBRs are strictly monotone only over the half-open interval $[0,1)$. To see that the average is not associative, consider AVER[AVER $(.8, .2), .4]=4.5 \neq 5.5=$ AVER[.8, AVER(.2,.4)]. As a consequence, AVPW cannot be associative.

The latter fact is relevant because, as will be seen, the intuitive appeal of AVPW as a subadditive metric with

Table 1

Summary of Formal Properties of Metrics $\mathbf{g}(\mathbf{a}, \mathbf{b})$

\begin{tabular}{lcccccc}
\hline Property & CB & SUP & EUC & MBR(k) & AVER & AVPW \\
\hline Additive & x & & & & & \\
Subadditive & & x & x & x & x & x \\
Monotone & x & x & x & x & & \\
Associative & x & x & & x & & \\
Bounded & & x & & x & x & x \\
Homogeneous & x & x & x & & x & \\
\hline
\end{tabular}


a built-in power transformation is supported by the relatively good fits we obtained with it. Presumably, its performance could be further enhanced by allowing the exponents to vary. However, we view its lack of monotonicity and associativity as a serious drawback. While concern for formal properties should not, of course, be allowed to override concern for the data, it strikes us as reasonable not to lose sight completely of mathematical tractability, and to select the most tractable metric when several fit equally well. From this point of view, the MBRs seem more attractive than the other metrics considered here. An added point in their favor is the ease with which they can be fitted to data: a simple inverse hyperbolic tangent transformation leads back to the cityblock metric. We now turn to the question of how well these functions fit the data.

\section{METHOD}

Nine solid light gray rectangles were presented in pairs against a dark background on the screen of a Radio Shack TRS-80 to 35 subjects. The stimulus design was completely crossed, $3 \times 3$, in the height/width coordinate system. The two rectangles of each pair $(x, y)$ appeared side by side. The widths were $2.7,5.4$, and $8.1 \mathrm{~cm}$, and the heights, $1.1,2.1$, and $3.1 \mathrm{~cm}$. The presentation sequence was random. All pairings were presented once in the order $(y, x)$ and once in the order $(x, y)$, except for the $(x, x)$ pairs, which were not presented.

The computer instructed the subject to rate the dissimilarity of each pairing on a scale from 0 (identical) to 9 (maximally different), and to enter each rating on the keyboard. Hitting the return key caused the next pairing to be displayed. The rating scale was also displayed on top of the monitor. To familiarize the subjects with the procedure and also with the stimulus range, the experimenter first exposed a sequence of 10 pairings in rapid succession and then requested the subject to complete 20 more pairings by entering his or her ratings. At this point, the subject was asked whether he or she had any questions. The program was then reset to initiate the actual experimental session, which usually took between 15 and 25 min.

The subjects were unpaid volunteers of both sexes in roughly equal distribution, ranging in age from 10 to 60 years, but otherwise unselected. No records were kept of the identities, ages, or sexes of the subjects.

\section{RESULTS}

\section{Individual Differences}

As a preliminary check, the individual data were analyzed with COSPA (Schönemann et al., 1978) in two dimensions. The plots suggested systematic individual diferences pointing to three different judgment strategies. Most subjects appeared to use height and width equally, a smaller subset of subjects mainly shape, and a still smaller subset mainly area. To discriminate among these three groups, we computed the difference between the lengths of the two main diagonals of the design $[d(3,7)-d(1,9]$. This function can be viewed as an orthogonality index. If it is close to zero, the subjective configuration is close to a square and the subject used height and width roughly equally (equal group). If it is positive, then the configuration is rhomboid, slanted toward the shape diagonal (up group). If it is negative, the config- uration is slanted toward the area diagonal (down group). The subjects were classified into one of these three groups, depending on whether this discriminant was larger than 1.5 (up group, 9 subjects), less than -1.5 (down group, 6 subjects), or between these two values (equal group, 20 subjects).

A multivariate analysis of variance on the diagonals of the eight remaining smaller squares as dependent variables confirmed that these strategy differences were consistent across all other (i.e., smaller) diagonal comparisons for the three groups $\left[\chi^{2}(16)=56.021, p<.000001\right.$, largest root theta $=.819]$. We therefore analyzed the individual data for each group separately. Since there were 20 subjects in the equal group, this group was split randomly into two subgroups to serve as a check on the consistency and robustness of our numerical results, which thus will be presented for four separate groups, labeled $\mathrm{E} 1, \mathrm{E} 2, \mathrm{UP}$, and DN.

\section{Metric Problem}

For all subjects in each group, the fit of the various metrics defined above was evaluated for all possible collinear comparisons parallel to the objective $\mathrm{H} / \mathrm{W}$ system and along both main diagonals, and for all possible triangular triples in terms of raw residuals, $e=g(a, b)-c$, and relative residuals, $e^{*}=[g(a, b)-c] /(a+b)$, where $\mathrm{g}(\mathrm{a}, \mathrm{b})$ is one of the nine functions defined earlier, and $a, b$, c are relative dissimilarities (observed dissimilarities divided by 9.1) corresponding to the notation in Figure 1. Although most results differed only negligibly for these two error measures, we will need the relative error later for comparisons across different stimulus configurations (e.g., across large and small triangles) for use in an attempt to answer the orientation question. Note that the raw residual e coincides for the $\mathrm{CB}$ with the function $f=a+b-c$ (Equation 1). Hence, for collinear comparisons, positive residual means indicate subadditivity and negative residual means indicate violations of the triangle inequality.

The main results of these direct within-subject analyses are summarized in Tables $2-4$. Since all observed ratings were divided by 9.1 ( 9 was the highest possible response), the analyzed values always varied over the half-open interval $[0,1)$. The residual means (Tables 2 and 3 ) provide an indication of the bias of the various metrics $g(a, b)$ toward super- or subadditivity for the collinear comparisons. The means are near zero for CB if, and only if, the data are additive. The means for any of the other metrics $\mathrm{g}(\mathrm{a}, \mathrm{b})$ are near zero if $\mathrm{g}(\mathrm{a}, \mathrm{b})$ restores collinear additivity. The residual sums of squares (SSQ, Table 4 ) indicate the fit of the various metrics $g(a, b)$ with smaller values corresponding to better fit.

\section{Bias}

The first three subdivisions in Table 2 assess the fit for segmental additivity parallel to the $\mathrm{H} / \mathrm{W}$ design coordinate system, and along the diagonals in terms of raw residuals. For the convenience of the reader, the bias results across all four groups are summarized in Table 3. 
Table 2

Bias: Means of Residuals $\mathbf{e}=\mathbf{g}(\mathbf{a}, \mathbf{b})-\mathbf{c}$

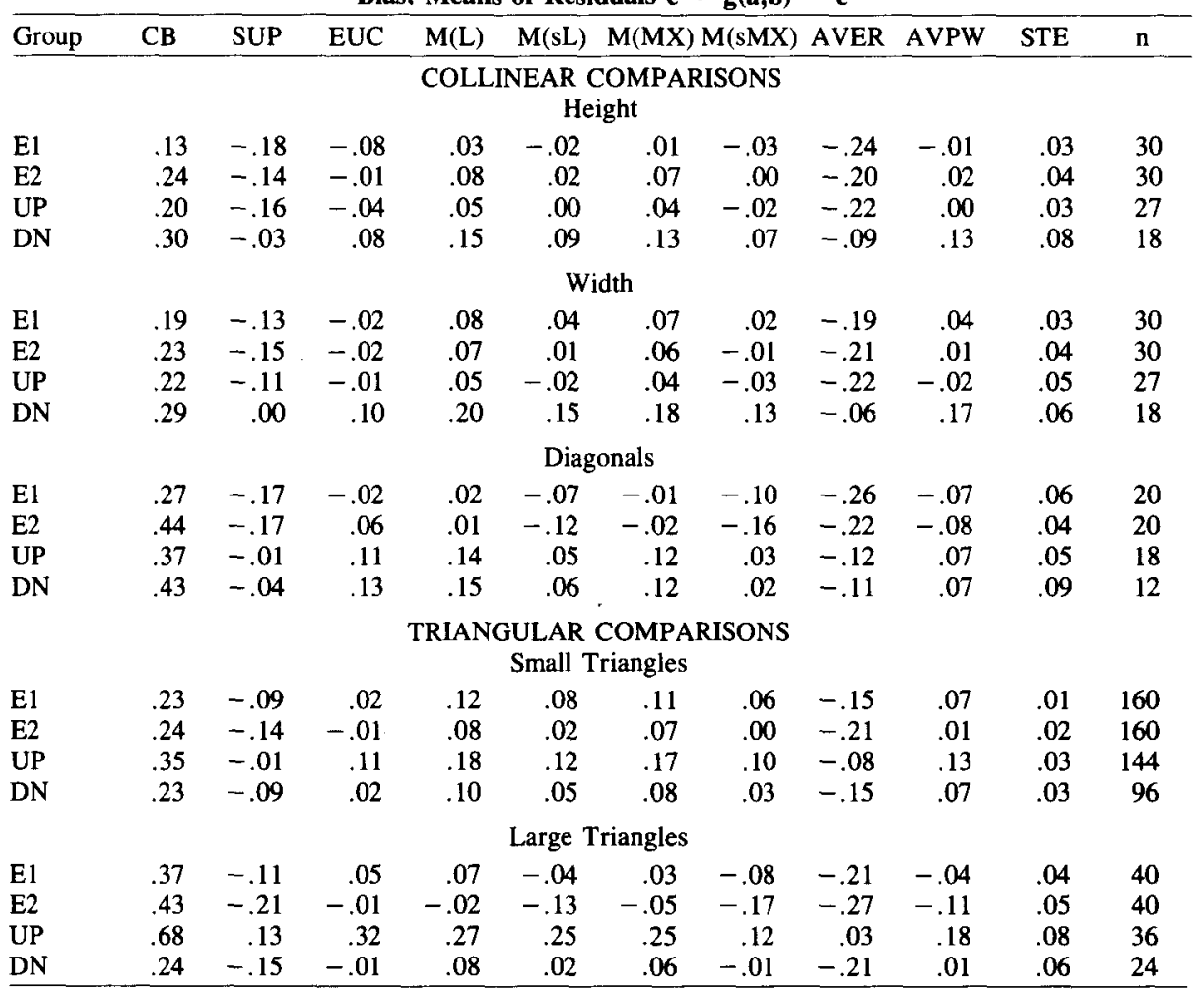

Table 3

Summary of Bias Patterns Based on Residual Means for Collinear Comparisons

\begin{tabular}{|c|c|c|c|c|c|c|c|c|c|}
\hline Group & CB & SUP & EUC & $M(L)$ & $\mathrm{M}(\mathrm{sL})$ & $\mathrm{M}(\mathrm{MX})$ & $\mathbf{M}(\mathrm{sMX})$ & AVER & AVPW \\
\hline \multicolumn{10}{|c|}{ HEIGHT } \\
\hline $\mathrm{E} 1$ & + & - & & & & & & - & \\
\hline E2 & + & - & & & & & & - & \\
\hline UP & + & - & & & & & & - & \\
\hline DN & + & & & & & + & & & + \\
\hline \multicolumn{10}{|c|}{ WIDTH } \\
\hline E1 & + & - & & & & & & - & \\
\hline $\mathrm{E} 2$ & + & - & & & & & & - & \\
\hline UP & + & - & & & & & & - & \\
\hline DN & + & & + & + & + & + & & & + \\
\hline \multicolumn{10}{|c|}{ DIAGONALS } \\
\hline E1 & + & - & & & & & - & - & \\
\hline E2 & + & - & & & & & - & - & \\
\hline UP & + & & + & + & & + & & - & \\
\hline DN & + & & + & + & & + & & - & \\
\hline
\end{tabular}

Note: - , mean $<.1$ ( $g$ too subadditive for data); blank, $-.1 \leq$ mean $\leq .1$ (g restores additivity); + , mean $>.1$ ( $g$ not subadditive enough for data). 
Table 4

Fit: Residual SSQ Relative to SSQ Left by CD [SSCB]

\begin{tabular}{|c|c|c|c|c|c|c|c|c|c|c|c|}
\hline Group & CB & SUP & EUC & $\mathrm{M}(\mathrm{L})$ & $\mathrm{M}(\mathrm{sL})$ & M(MX & $\mathrm{M}(\mathrm{sMX})$ & AVER & AVPW & [SSCB] & $\mathbf{n}$ \\
\hline \multicolumn{12}{|c|}{$\begin{array}{c}\text { COLLINEAR COMPARISONS } \\
\text { Height }\end{array}$} \\
\hline E1 & 1. & 1.01 & .50 & .37 & .33 & .36 & .35 & 1.52 & .31 & {$[.05]$} & 30 \\
\hline $\mathrm{E} 2$ & 1. & .48 & .31 & .34 & .27 & .30 & .23 & .63 & .24 & [.11] & 30 \\
\hline UP & 1. & .61 & .23 & .24 & .25 & .22 & .26 & .97 & .28 & {$[.06]$} & 27 \\
\hline $\mathrm{DN}$ & 1. & .19 & .29 & .28 & .16 & .22 & .12 & .18 & .19 & [.19] & 18 \\
\hline \multicolumn{12}{|c|}{ Width } \\
\hline $\mathrm{E} 1$ & 1. & .61 & .36 & .43 & .32 & .41 & .33 & .88 & .34 & {$[.07]$} & 30 \\
\hline $\mathrm{E} 2$ & 1. & .45 & .32 & .33 & .27 & .32 & .28 & .66 & .25 & {$[.11]$} & 30 \\
\hline UP & 1. & .28 & .24 & .22 & .19 & .20 & .19 & .62 & .19 & {$[.11]$} & 27 \\
\hline DN & 1. & .15 & .29 & .42 & .27 & .35 & .21 & .14 & .28 & [.15] & 18 \\
\hline \multicolumn{12}{|c|}{ Diagonals } \\
\hline E1 & 1. & .52 & .35 & .23 & .24 & .22 & .26 & .65 & .21 & {$[.16]$} & 20 \\
\hline E2 & 1. & .17 & .15 & .04 & .08 & .03 & .11 & .25 & .06 & {$[.27]$} & 20 \\
\hline UP & 1. & .12 & .19 & .23 & .20 & .22 & .21 & .23 & .22 & [.19] & 18 \\
\hline DN & 1. & .16 & .27 & .25 & .17 & .22 & .16 & .23 & .20 & [.29] & 12 \\
\hline \multicolumn{12}{|c|}{$\begin{array}{c}\text { TRIANGULAR COMPARISONS } \\
\text { Small Triangles }\end{array}$} \\
\hline E1 & 1. & .35 & .27 & .42 & .31 & .36 & .25 & .51 & .31 & [.09] & 160 \\
\hline $\mathrm{E} 2$ & 1. & .45 & .33 & .32 & .24 & .29 & .22 & .62 & .22 & {$[.12]$} & 160 \\
\hline UP & 1. & .24 & .35 & .38 & .27 & .36 & .26 & .26 & .29 & {$[.22]$} & 144 \\
\hline $\mathrm{DN}$ & 1. & .37 & .40 & .36 & .25 & .31 & .22 & .42 & .27 & [.15] & 96 \\
\hline \multicolumn{12}{|c|}{ Large Triangles } \\
\hline E1 & 1. & .26 & .26 & .19 & .15 & .15 & .16 & .40 & .16 & {$[.21]$} & 40 \\
\hline E2 & 1. & .35 & .24 & .07 & .12 & .07 & .16 & .48 & .13 & [.22] & 40 \\
\hline UP & 1. & .17 & .37 & .25 & .16 & .23 & .15 & .19 & .21 & [.68] & 36 \\
\hline DN & 1. & .66 & .53 & .51 & .44 & .48 & .43 & .74 & .43 & {$[.15]$} & 24 \\
\hline
\end{tabular}

Note-The three best-fitting functions in each row are italicized.

For the city-block metric (second column in Table 2) the residual means are substantially positive for all collinear comparisons (first three blocks), averaging about $1 / 5$ of the response scale. Since all Minkowski metrics are additive within dimensions, this consistent finding of segmental subadditivity in all four directions rules out all Minkowski metrics for both a $\mathrm{H} / \mathrm{W}$ and an $\mathrm{A} / \mathrm{S}$ coordinate system. It also contradicts Tversky and Gati's (1982) coincidence hypothesis that "dissimilarities are additive across attributes and subadditive within attributes" (p. 134). Our dissimilarities are segmentally subadditive both ways.

In the case of a perfect fit, these means would be zero. This is approximately the case for the Euclidean metric, the four MBRs, and AVPW across the E1, E2, and UP groups. However, the means do not favor one orientation over the other.

Figure 3 depicts graphically the relative improvement of the fit over the CB obtained with two of the MBRs in terms of the cumulative error distributions. The medians for the $C B$ residuals fall consistently to the right of the $(-.1, .1)$ interval. For the two MBRs, the medians wander to the left and the ogives become steeper. This joint reduction in bias and variance combines to give a better fit.

The residual means are consistently negative for the sup metric and the average (AVER). This means both func- tions overcompensate for the subadditivity in the data so that the transformed data violate the triangle inequality.

As a rough conservative gauge for the magnitude of the residual means, the largest standard error per row (STE) is given in the next-to-last column of Table 2 . This measure was computed in the usual way. However, since the observations on which it is based are not independent, it has only descriptive, not inferential, significance. The median standard error was usually about half STE.

Since statistical tests are difficult to justify with such data containing within-subject dependencies, our inferences will rely on the consistency across the four independent subgroups of subjects. Perhaps we should add, in response to a reviewer, that we view replication quite generally as a preferable alternative to one-sample statistical tests. We agree with Guttman (1977) that "the essence of science is replication," not statistical inference. It is not hard to find data for which any preconceived model cannot be rejected as long as one sees to it that the statistical test is sufficiently weak in power. This consistency across independent replications, although perhaps already evident in Table 2 , is brought out more clearly in the bias pattern of the mean residuals in Table 3 .

Overall, then, it appears that the four MBRs, EUC, and AVPW exhibit the least additivity bias, and CB and AVER the most, in opposite directions, at least across the first three groups. Since the last group (DN) is the smallest, 


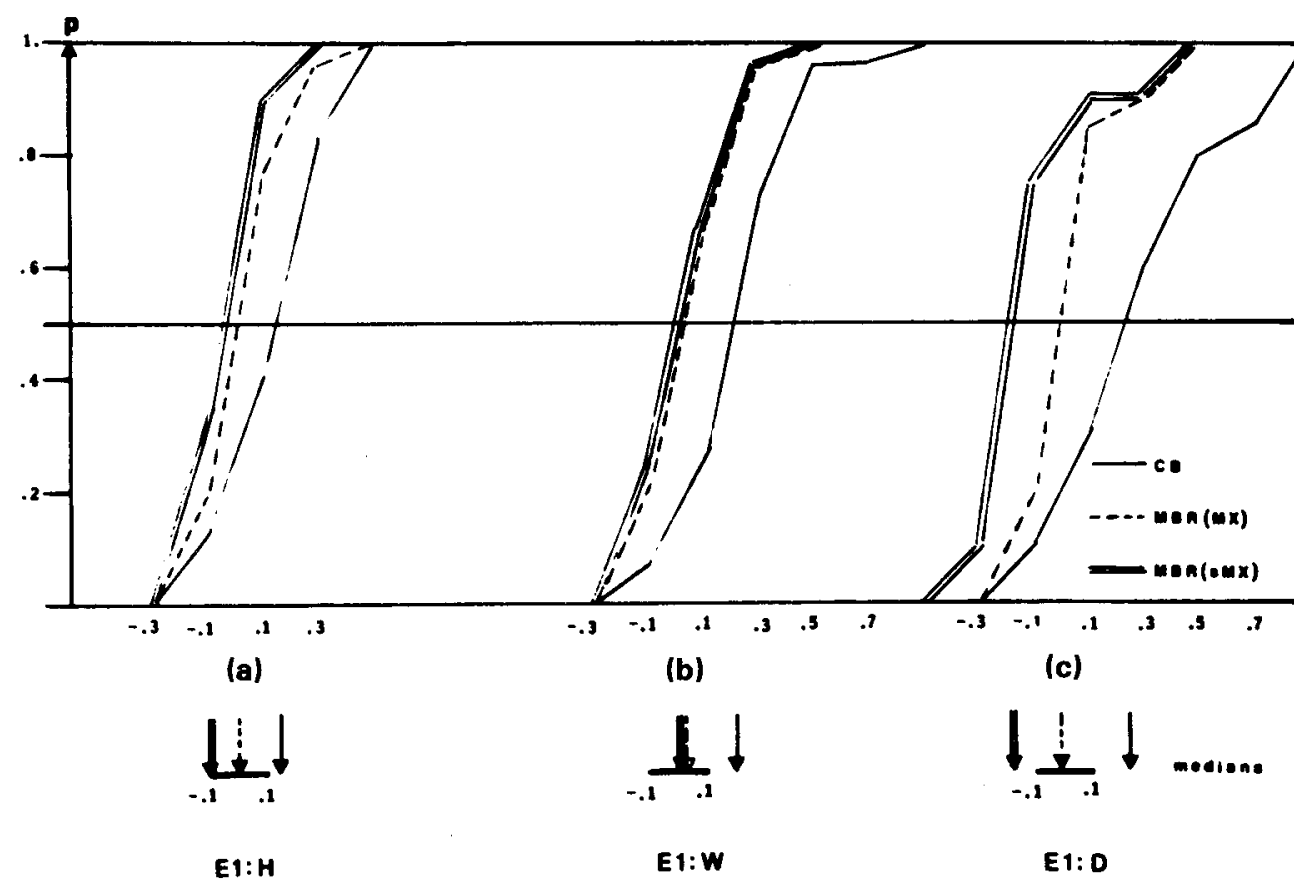

Figure 3. Restoration of segmental additivity by MBR(MX) and MBR(sMX) for the E1 group Imedians move left toward $(-1,1)$ interval]. Collinear comparisons (a) parallel to height, (b) parallel to width, and (c) along both diagonals.

with only six subjects, we are inclined to attach the least significance to the results obtained for it.

\section{Fit}

In Table 4, the average sums of squared residuals for each metric $g(a, b)$ are given relative to those left by the city-block metric. Since this ratio is in most cases $(99 \%)$ a proper fraction, the city-block metric usually fits much worse than any of the other metrics. The three best-fitting functions have been italicized in each row. The actual residual sum of squares for the city-block metric is given in the next-to-last column ([SSCB]). All other absolute SSQs can be found, if desired, by multiplying SSCB with the relative sums of squares in each row. However, for the purpose of comparing the fit of the various metrics $\mathrm{g}(\mathrm{a}, \mathrm{b})$, the relative SSQ given in the body of Table 4 should suffice. The last column (n) gives the number of triples involved in each SSQ.

The number of times each function placed among the three best-fitting functions for each comparison across all blocks is totaled in Part A of Table 6. One finds that MBR(sL) and MBR(sMX) (with $s=.8$ ) give the best fit overall. The city-block metric gives the worst fit overall. Of particular interest to us was the fact that the subjectspecific MBRs, MBR(MX) and MBR(sMX), did not, as expected, outperform the simple $M B R(L)$ and $M B R(s L)$, which are parameter-free (if we agree to always use $s=.8$, as we suggest). It is also of interest that AVPW fits only slightly less well than MBR(sL) and much better than MBR(L). In terms of fit alone, AVPW does surprisingly well, considering that it does not require any subject-specific adjustments, as do the $\mathrm{MBR}(\mathrm{MX})$ and MBR(sMX). As already noted, our main reservation against AVPW is its lack of monotonicity and associativity.

Since we did not find any systematic differences in fit between the height and width comparisons and the diagonal comparisons-the data are strongly subadditive both ways-we conclude, overall, that MBR(sL) and MBR(sMX) with $s=.8$ give the best fit to our data, regardless of whether the subjects use a H/W or an A/S system. Since the difference in fit between these two metrics is negligible, and MBR(sMX) involves an additional parameter, we recommend working with $\mathrm{MBR}(\mathrm{sL})$, with $s=.8$.

Table 5 shows that the results for the Borg and Leutner data are quite similar. Hence, the "ample evidence ... that the city-block metric is the adequate distance function" cited by Borg and Leutner (1983, p. 260) must be sought in data other than their own. For those the CB gives uniformly the worst fit among the three major Minkowski metrics, just as it did for our own data. The same is true for the ellipse data reported by Noma and Johnson (1977).

\section{Orientation Problem}

On the basis of the COSPA analyses and the orthogonality index, one expects different orientations in the different subgroups. In an attempt to assess the relative fit in the two coordinate systems quantitatively, we com- 
Table 5

Bias and Fit in Borg and Leutner (1981) Data:

For the Three Major Minkowski Metrics

\begin{tabular}{|c|c|c|c|c|c|c|c|}
\hline \multirow[b]{2}{*}{ Group } & \multicolumn{3}{|c|}{$\begin{array}{l}\text { BIAS: } \\
\text { Means of residuals } \mathrm{e}^{*}=\mathrm{g}(\mathrm{a}, \mathrm{b})-\mathrm{c}\end{array}$} & \multicolumn{4}{|c|}{$\begin{array}{l}\text { FIT: } \\
\text { Residual SSQ relative to that left by CB [SSCB] }\end{array}$} \\
\hline & $\mathrm{CB}$ & SUP & EUC & $\mathrm{CB}$ & SUP & EUC & [SSCB] \\
\hline \multicolumn{8}{|c|}{$\begin{array}{c}\text { COLLINEAR COMPARISONS } \\
\text { Height }\end{array}$} \\
\hline $\mathrm{H} / \mathrm{W}$ & .27 & -.15 & .00 & 1. & .32 & .01 & {$[.07]$} \\
\hline $\mathrm{A} / \mathrm{S}$ & .19 & .01 & .00 & 1. & .34 & .08 & {$[.04]$} \\
\hline \multicolumn{8}{|c|}{ Width } \\
\hline $\mathrm{H} / \mathrm{W}$ & .14 & -.09 & -.01 & 1. & .42 & .03 & {$[.02]$} \\
\hline $\mathrm{A} / \mathrm{S}$ & .27 & -.15 & -.01 & 1. & .32 & .03 & {$[.08]$} \\
\hline \multicolumn{8}{|c|}{ Diagonals } \\
\hline $\mathrm{H} / \mathrm{W}$ & .30 & -.18 & -.02 & 1. & .37 & .04 & {$[.10]$} \\
\hline $\mathrm{A} / \mathrm{S}$ & .23 & .19 & .05 & 1. & .66 & .06 & {$[.06]$} \\
\hline \multicolumn{8}{|c|}{$\begin{array}{l}\text { TRIANGULAR COMPARISONS } \\
\text { Small Triangles }\end{array}$} \\
\hline $\mathrm{H} / \mathrm{W}$ & .17 & -.08 & -.01 & 1. & .35 & .11 & {$[.03]$} \\
\hline $\mathrm{A} / \mathrm{S}$ & .33 & .00 & .11 & 1. & .04 & .15 & {$[.11]$} \\
\hline \multicolumn{8}{|c|}{ Large Triangles } \\
\hline $\mathrm{H} / \mathrm{W}$ & .36 & -.15 & .10 & 1. & .14 & .02 & {$[.14]$} \\
\hline $\mathrm{A} / \mathrm{S}$ & .41 & -.08 & .06 & 1. & .11 & .10 & {$[.20]$} \\
\hline
\end{tabular}

puted the relative residuals $\mathrm{e}^{*}$ for two subsets of stimuli: a larger square " $\mathrm{HW}$ " defined by the outer vertices of the design square, with sides parallel to height and width (defined by stimuli $1,3,7,9$ ), and a smaller rotated square "AS" (defined by stimuli 2, 4, 6, 8) whose sides are roughly parallel to an A/S system-only roughly because our physical design employed metric rather than logged height and width.

Now let $\mathrm{SS}(\mathrm{HW})$ be the sum of squared relative residuals $e^{*}=[g(a, b)-c] /(a+b)$, where $c$ is the observed diagonal rating and $a, b$ are the ratings of the two adjacent sides. Since there are two diagonals and two pairs of adjacent sides to each diagonal, $\mathrm{SS}(\mathrm{HW})$ is based on four residuals, with an analogous definition of SS(AS). The fit is based on a comparison of the relative errors $\mathrm{e}^{*}$ to compensate for the size of the two squares. To discriminate between the fit in both directions (either parallel to $\mathrm{H} / \mathrm{W}$ or parallel to $\mathrm{A} / \mathrm{S}$ ) we compute the discriminator

$$
q=[S S(A S)-S S(H W)] /[S S(A S)+S S(H W)]
$$

which varies between -1 and 1 . If it is positive, it favors a $\mathrm{H} / \mathrm{W}$ system (because it leaves a smaller sum of squares than an A/S shape system); if it is negative, it favors an $\mathrm{A} / \mathrm{S}$ system; and if it is close to zero, this discriminator leaves the orientation question open. Given our complete lack of experience with this function, the small number of data points which enter it, and the absence of any norms, it seems wise to limit the interpretation to reasonably large values of $q$, perhaps on the order of .2 or .3 in magnitude. The values for $q$ are summarized in Part $B$ of Table 6 for all four groups. The metrics $g(a, b)$ have been reordered to correspond to the order of fit (Part A), with the best-fitting metrics on the left and the worst-fitting on the right.
On the basis of this rough criterion, the city-block metric appears to favor the $\mathrm{H} / \mathrm{W}$ system for the two $\mathrm{E}$ groups and the DN group, but to favor the A/S system for the UP group. These trends are as expected, except for the DN group, which is small and probably untrustworthy. The MBR(L) and the MBR(MX) show the same tendency as the CB function. However, the three bestfitting metrics-MBR(sL), MBR(sMX), and AVPW - seem to favor the A/S system mildly but consistently for the two equal groups, contrary to expectations. It thus seems fair to say that an answer to the orientation question may be less clear-cut than the earlier quote from Borg and Leutner (1983) suggests. However, in view of the tenuous nature of our index $q$, especially the relatively small number of points on which it is based, it is perhaps best to defer judgment on the orientation question. We will return to it in the next section, in which we present the MDS analyses of the group-averaged data.

\section{Multidimensional Scaling Analyses of Group Averages}

The relative dissimilarity data were averaged within three groups $(\mathrm{EQ}=\mathrm{E} 1+\mathrm{E} 2, \mathrm{UP}, \mathrm{DN}$; see Table 7) and then analyzed with MRSCAL (Roskam, 1972). This is an iterative program for fitting the city-block metric (and all other Minkowski metrics) under known monotone transformations. In our case, this was the identity transformation.

The results for our initial runs with the starting configuration provided by the program were disappointing for the pooled EQ group ( 20 subjects). The residual sum of squares was surprisingly large $(.306$, with 10 residuals exceeding . 1 on a scale from 0 to 1 ), the sides of the configuration were strongly curved, and its position seemed to favor $\mathrm{A} / \mathrm{S}$, contrary to expectations. Inspection of the 
Table 6

Orientation Problem

\begin{tabular}{lccccccccc}
\hline \multicolumn{7}{c}{ A. Frequency With Which Each Metric $\mathrm{g}(\mathrm{a}, \mathrm{b})$} & Placed Among Three Best-Fitting Metrics* \\
\hline M(sL) & $\mathrm{M}(\mathrm{sMX})$ & AVPW & M(MX) & SUP & M(L) & AVER & EUC & CB & $\begin{array}{c}\text { [group } \\
\text { size] }\end{array}$ \\
\hline 15 & 14.5 & 10.5 & 5 & 5 & 4 & 3 & 3 & 0 & [35] \\
\hline
\end{tabular}

B. Values of Orientation Index $q=[\operatorname{SSQ}(\mathrm{AS})-\mathrm{SSQ}(\mathrm{HW})] /[\mathrm{SSQ}(\mathrm{AS})+\mathrm{SSQ}(\mathrm{HW})]^{* *}$

\begin{tabular}{lcccccccccc}
\hline Group & M(sL) & M(sMX) & AVPW & M(MX) & SUP & M(L) & AVER & EUC & CB & $\begin{array}{c}\text { [group } \\
\text { size] }\end{array}$ \\
\hline E1 & -.06 & -.15 & -.03 & .24 & -.33 & .22 & -.37 & .01 & .22 & {$[10]$} \\
E2 & -.14 & -.31 & -.08 & .35 & -.45 & .45 & -.48 & .15 & .42 & [10] \\
UP & -.23 & -.25 & -.45 & -.23 & -.15 & -.21 & -.26 & -.38 & -.29 & {$[9]$} \\
DN & .06 & -.06 & .05 & .15 & -.64 & .20 & -.78 & -.07 & .34 & {$[6]$} \\
\hline
\end{tabular}

C. Average Residual SSQ Left by (two-dimensional) MDS $\dagger$

\begin{tabular}{|c|c|c|c|c|c|}
\hline \multirow[b]{2}{*}{ Group } & \multirow{2}{*}{$\begin{array}{c}\text { Starting } \\
\text { Configuration }\end{array}$} & \multicolumn{3}{|c|}{ Metrics $g(a, b)$} & \multirow{2}{*}{$\begin{array}{c}\text { [group } \\
\text { size] }\end{array}$} \\
\hline & & $\mathrm{CB}$ & $\operatorname{MBR}(L)$ & MBR(sL) & \\
\hline $\begin{array}{l}\mathrm{E} 1+\mathrm{E} 2 \\
(=\mathrm{EQ})\end{array}$ & $\begin{array}{l}\mathrm{H} / \mathrm{W} \\
\mathrm{A} / \mathrm{S}\end{array}$ & $\begin{array}{l}.006 \\
.010\end{array}$ & $\begin{array}{l}.002 \\
.006\end{array}$ & $\begin{array}{l}.004 \\
.009\end{array}$ & [20] \\
\hline UP & $\begin{array}{l}\mathrm{H} / \mathrm{W} \\
\mathrm{A} / \mathrm{S}\end{array}$ & $\begin{array}{l}.011 \\
.006\end{array}$ & $\begin{array}{l}.003 \\
.003\end{array}$ & $\begin{array}{l}.006 \\
.010\end{array}$ & [9] \\
\hline DN & $\begin{array}{l}\text { H/W } \\
\mathbf{A} / \mathrm{S}\end{array}$ & $\begin{array}{l}.009 \\
.014\end{array}$ & $\begin{array}{l}.006 \\
.012\end{array}$ & $\begin{array}{l}.010 \\
.017\end{array}$ & [6] \\
\hline
\end{tabular}

*Across all four groups and five comparisons (see Table 4). **Positive values favor $\mathrm{H} / \mathrm{W}$, negative values A/S system. †The best-fitting solutions across starting configurations are italicized.

averaged data revealed that the inverse hyperbolic tangent transformation had restored additivity along the $\mathrm{H} / \mathrm{W}$ dimensions to a much larger extent than this MDS solution indicated. When we computed a second solution directly from the transformed data, the residual sum of squares was reduced by three-fourths (from .306 to .073), with only one discrepancy above .1 . There seemed little doubt that the program had become trapped in a local minimum. The better-fitting solution was virtually veridical in a $\mathrm{H} / \mathrm{W}$ system and free from any interactions. The solution for the UP group pointed toward shape as the dominant dimension, and the solution for the (rather small) DN group favored area as the dominant dimension. Since these earlier MDS configurations have already been published in Schönemann and Kienapple (1984), they are not reproduced here.

Our more recently completed within-subject analyses, reported above, suggest that the fit of the MBR could be enhanced by reducing the upper bound by about a fifth of the response range [MBR(sL) with $s=.8$ ]. Although the results for the three-group averages were not identical to those obtained at the individual level (Tables 2-4), the overall trends were very similar. They can be summarized as follows: For the averaged data, the MBR(sMX) gave the best overall fit for the group data, placing 13 times among the best 3; MBR(L) placed 3 times and MBR(sL) placed 5 times. This discrepancy with the individual analyses [in which MBR(sL) and MBR(sMX) did equally well] is only apparent, because the differences in residual sums of squares were quite small. The CB gave again the worst fit across all comparisons and all groups.
Since we tend to trust the individual data more than the group averages, we decided to fit the MDS solutions to the averaged group data for three parameter-free metrics, the CB metric, the MBR(L) with fixed upper bound, and the $\mathrm{MBR}(\mathrm{sL})$ with reduced fixed upper bound $(\mathrm{sL}=.8 \mathrm{~L}$ $=7.2)^{3}$

Forewarned by our earlier experiences with random starting configurations, we provided two external start-

Table 7

Group Averages of Observed Dissimilarities

EQ Group $(\mathrm{N}=20)$ Below Diagonal

UP Group $(\mathrm{N}=9)$ Above Diagonal

$\begin{array}{lllllllll}.000 & .358 & .475 & .543 & .382 & .370 & .789 & .475 & .339\end{array}$

$\begin{array}{lllllllll}.388 & .000 & .302 & .789 & .388 & .345 & .869 & .555 & .432\end{array}$

$\begin{array}{lllllllll}.491 & .305 & .000 & .845 & .518 & .382 & .882 & .703 & .536\end{array}$

$\begin{array}{lllllllll}.405 & .660 & .802 & .000 & .610 & .746 & .432 & .388 & .728\end{array}$

$\begin{array}{lllllllll}.613 & .466 & .655 & .508 & .000 & .333 & .814 & .388 & .259\end{array}$

$\begin{array}{lllllllll}.771 & .527 & .369 & .669 & .397 & .000 & .888 & .567 & .339\end{array}$

$\begin{array}{lllllllll}.649 & .749 & .849 & .358 & .594 & .777 & .000 & .647 & .777\end{array}$

$\begin{array}{lllllllll}.769 & .630 & .777 & .583 & .447 & .608 & .474 & .000 & .382\end{array}$

$\begin{array}{lllllllll}.835 & .752 & .585 & .757 & .530 & .369 & .660 & .377 & .000\end{array}$

DN Group $(\mathrm{N}=6)$

.000

$.296 \quad .000$

$\begin{array}{lll}.388 & .287 & .000\end{array}$

$\begin{array}{llll}.379 & .527 & .564 & .000\end{array}$

$\begin{array}{lllll}.675 & .361 & .499 & .351 & .000\end{array}$

$\begin{array}{llllll}.786 & .481 & .324 & .379 & .342 & .000\end{array}$

$\begin{array}{lllllll}.518 & .536 & .462 & .361 & .416 & .518 & .000\end{array}$

$\begin{array}{llllllll}.758 & .481 & .546 & .527 & .506 & .481 & .370 & .000\end{array}$

\begin{tabular}{lllllllll}
.777 & .601 & .416 & .666 & .573 & .379 & .453 & .453 & .000 \\
\hline
\end{tabular}

Note-Italicized figures indicate comparisons $d(I, 9)$ and $d(3,7)$ which were used to classify subjects into subgroups. 
ing configurations, one for $\mathrm{H} / \mathrm{W}$ and one for $\mathrm{A} / \mathrm{S}$, for each group, to counteract the dependency of the results on the starting configuration (see also below).

The residual sums of squares of the 18 MDS solutions ( 3 groups $\times$ metrics $\times 2$ starting configurations) are shown in Part $\mathrm{C}$ of Table 6, in which the best-fitting solutions across starting configurations are italicized. The worst-fitting group is the DN group, and the worst-fitting metric is the CB. The best-fitting metric in this case is $\operatorname{MBR}(L)$, not MBR(sL), which gave the best fit for the within-subject analyses. Both MBRs fit about two or three times better than the additive CB for the two larger groups.

The most striking feature of Table 6, Part C, is the extent to which the fit depends on the starting configuration, which, ideally, should not affect the fit upon convergence. Borg and Leutner (1983, p. 16) report similar experiences with KYST. This suggests that these difficulties are not restricted to MRSCAL but reflect the limitations of such iterative algorithms generally. To counteract this bias, it may be prudent to initialize the runs with both starting configurations, $\mathrm{H} / \mathrm{W}$ and $\mathrm{A} / \mathrm{S}$, before making any inferences about the orientation question.

More generally, this dependency on the starting con- figuration further tempers our confidence in the results obtained with such iterative MDS programs, including those reported by Borg and Leutner (1983), when it comes to answering nontrivial questions, such as the metric question or the orientation question. It will be recalled that these authors concluded that the city-block metric described their averaged data best and that the orientation was $\mathrm{H} / \mathrm{W}$. However, they presented no specific evidence for the city-block metric-they simply relied on previous "ample evidence ... that the city-block metric is the adequate distance function for 'analyzable' stimuli such as rectangles" (p. 260). Since our reanalysis of their data (Table 5) leaves no doubt that the city-block metric actually gave the worst fit of the three major Minkowski metrics, just as it did for our data, it appears rather risky to rely on this particular metric for inferences about the orientation problem.

For our own averaged data, the best-fitting solutions favor the H/W system for the EQ group (Figure 4) and the DN group (not shown), and an $\mathrm{A} / \mathrm{S}$ system for the UP group (Figure 5), with a strong dominance of shape over area. In agreement with the MBR hypothesis, initially pronounced curvilinear distortions of the $\mathrm{CB}$ solutions are
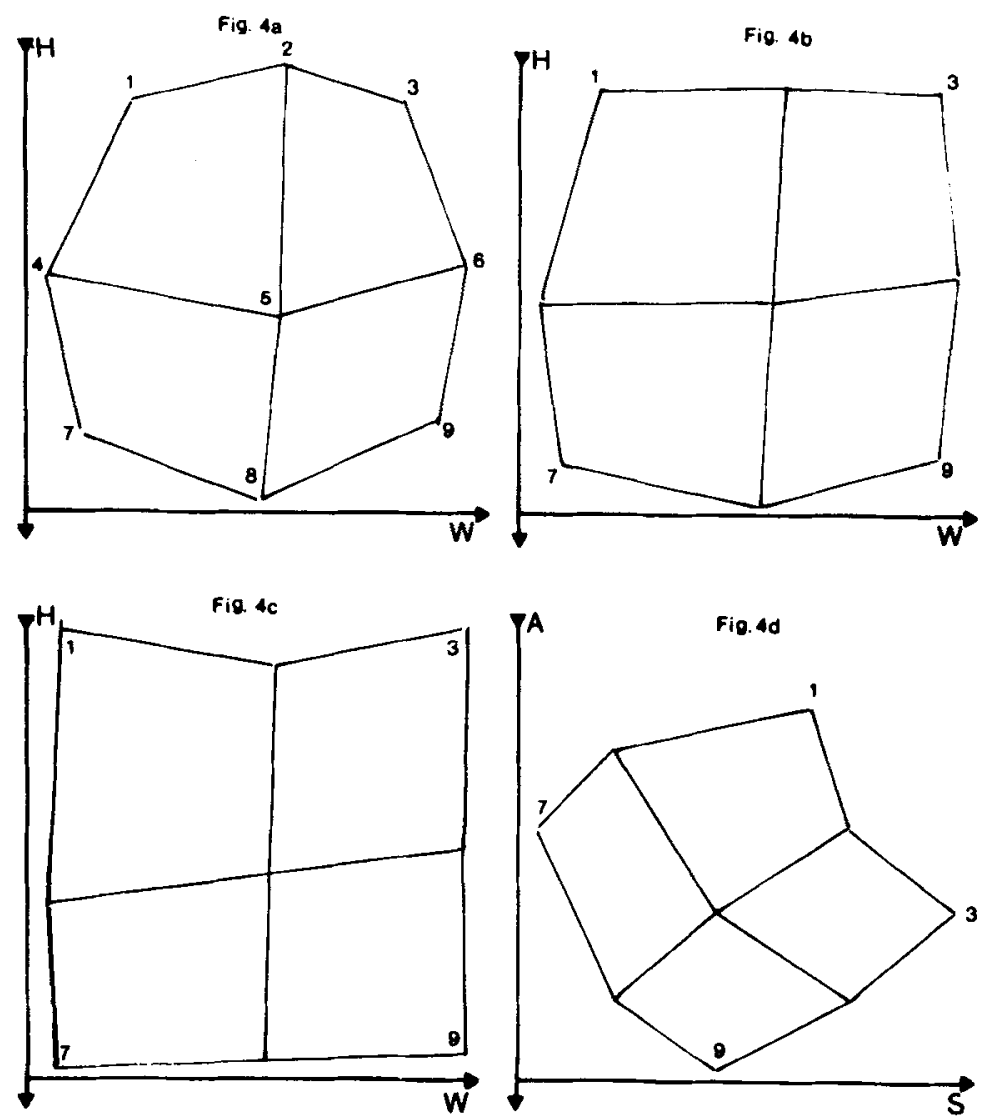

Figure 4. MRSCAL solutions for different metrics and starting configurations, EQ groups (averaged data, $N=20$ ). (a) $C B$ metric, $H / W$; note curvature. (b) $M B R(L)$, H/W. (c) MBR(sL), H/W. (d) MBR(sL), A/S; "wrong" starting configuration. 



Figure 5. MRSCAL solutions for different metrics and starting configurations, UP group (averaged data, $N=9$ ). (a) CB metric, A/S. (b) MBR(L), A/S. (c) MBR(sL), A/S; shape effect most pronounced. (d) MBR(sL), H/W; "wrong” starting configuration.

progressively straightened out in the two MBR solutions. For the EQ group, the MBR(sL) solution is virtually identical with the design. In particular, there are no signs of any $\mathrm{A} / \mathrm{S}$ interactions.

Finally, it should be noted that the configurations in Figures 4 and 5 are those produced by the MRSCAL program, that is, in terms of the coordinate projections $u, v$ in Equation 6, rather than a,b, which would result after applying the $\tan h$ transformation to $u, v$. Since the $\tan h$ transformation approximates the identity transformation over the lower range $[0, .4]$, this last step would change the shape of the configurations very little. Had there been pronounced logarithmic contraction effects along the coordinate axes, then the final tanh transformation would further reduce them, because it contracts larger projections more strongly than smaller ones.

The adjustments needed to adapt the MRSCAL algorithm to MBR(sL) and MBR(sMX) could have been circumvented with the construction of a least squares algorithm which fits the MBR(sL) directly. However, at this point, our primary objective was to demonstrate that the subadditive MBRs give a better fit overall to the observed data than that given by the city-block metric, and that the basic predictions of the theory from which the
MBR was derived replicate across all three groups. We obtained a better fit with the city-block metric after the inverse hyperbolic tangent transformation than for the observed data, curvilinear distortions were straightened out, and the subjective configurations confirmed the results of the within-subject analyses reported above, at least for the two larger EQ and UP groups.

\section{DISCUSSION}

We found converging evidence, based on six different data sets (the two groups of the Borg and Leutner study and the four subgroups of our own experiment), that subjects tend to produce subadditive dissimilarity judgments when rating pairs of rectangles on a category scale. This pervasive subadditivity effect may have been overlooked in the past because the mechanical interpolation of monotone transformations between data and reproduced distances tends to obscure it. When Attneave (1950) was confronted with the same effect, he attached no significance to it.

In contrast, we believe this consistent subadditivity in dissimilarity ratings calls for an explanation. We have tried to give such an explanation with our MBR hypothe- 
sis. Our detailed within-subject analyses leave no room for doubt that this hypothesis accounts for the individual observed data better than any of the Minkowski metrics, and also better than the other subadditive metrics we have investigated here. We feel the fact that these findings replicated across four independent subgroups, and also to a large extent across different studies, is better proof of their trustworthiness than any one-sample statistical test, quite apart from the within-subject dependencies and the power problem.

Our data did not confirm the interaction effect reported by Krantz and Tversky (1975) or the coincidence hypothesis advanced by Tversky and Gati (1982). Our reanalysis also casts some doubt on the conclusions Borg and Leutner (1983) derived from their grouped data on the basis of MDS analyses.

We found consistent individual differences pointing toward different judgment strategies and leading to distinct MDS configurations in the respective subgroups. This is in accord with our basic premise that the question of how subjects perceive rectangles probably cannot be answered once and for all. We do not view the production of dissimilarity ratings as a perceptual problem that is ultimately reducible to sensory mechanisms, but rather as a primarily cognitive task akin to concept formation. From this point of view, it is not surprising that different subjects should employ different strategies (e.g., Gigerenzer, 1983), or that their strategies change when the task or stimulus material is changed.

The search for the appropriate psychophysical law proved futile because the mathematical form of the response depends on many things-the subject population, the instruction and the standard, the context, the stimulus range, and the response range (e.g., Baird \& Noma, 1978; Birnbaum, 1982; Engen \& Levy, 1955; Parducci, 1982; Poulton, 1968; M. Teghtsoonian \& R. Teghtsoonian, 1971; R. Teghtsoonian, 1971).

For similar reasons, the search for the appropriate metric may also be futile. Rather than trying to impose on the data some arbitrarily selected metric with a currently fashionable program, the real challenge, we believe, is to determine (1) whether the data can be described by any metric at all, (2) if so, what this metric might be, and (3) what psychological mechanisms might explain it.

These questions cannot be answered once monotone transformations are mechanically interpolated between data and model. The first question can no longer be answered because any data set can be deformed into a metric by a suitably chosen monotone transformation. The second question is made much more difficult because monotone transformations interact with metrics. Without an answer to the first two, we cannot hope to answer the third question. We therefore consider it more promising to try to answer these questions on the basis of the actually observed data and to justify any transformation of the data in psychological terms.

A reviewer has asked us to elaborate on this position and to contrast the process approach advocated here with the so-called "representational measurement" point of view. Broadly speaking, we view the latter as an optimal outcome of the former. In other words, we believe formalized models are a desirable end result of, not a prerequisite for, productive empirical research. For example, to start with the assumption that subjects use a spatial representation when making dissimilarity judgments may be premature. What we initially should look for is not confirmation of some preconceived formal model, but rather nontrivial empirical relations which stand up under repeated replication, and preferably also under changing experimental conditions (Birnbaum, 1982), which may suggest a model worth formalizing.

At this point, we entertain little doubt that the subadditivity effect in dissimilarity ratings on bounded response scales is replicable and pervasive. We are much less certain how it comes about. Our MBR hypothesis is one possible explanation, but certainly not the only one. Professor W. Tack of the University of Saarbrucken (personal communication, 1984) has suggested that subadditivity might be due to sampling error: If one assumes independence of judgmental processes and homogeneous error variances, then it follows (e.g., within the framework of Thurstone's law of comparative judgment) that the sum of two contiguous collinear ratings exceeds the rating of the sum. A third possible explanation might be a general tendency toward conservative judgments, a cognitive "regression to the mean" effect, so to speak.

However, regardless of which perceptual and cognitive processes produce the segmental subadditivity, it does cast some doubt on the popular notion of a "cognitive map," an internal spatial representation of the stimuli somehow invoked by subjects when they produce dissimilarity ratings. The outcome of MDS analyses, it is hoped, may provide an isomorphic image of this cognitive map. However, if the data are subadditive as a result of the decision process the subject invokes to rate pairs of stimuli-which is all the subject is asked to do-then the data will automatically satisfy the requirements of a metric (in particular, the triangle inequality). We then have a good chance of approximating the data with some finite-dimensional metric so as to obtain a spatial representation, whether the subjects actually use it or not. In other words, the integration of local (i.e., pairwise) information into a global spatial representation may actually be performed by the experimenter (and his program), instead of by the subjects. In this case, the presumed cognitive map would not tell us much about the subjects, and, as an artifact of our research strategies, it might actually deflect attention from the problem we set out to study.

\section{REFERENCES}

Anderson, N. H. (1970). Functional measurement and psychophysical judgement. Psychological Review, 77, 153-170.

Atrneave, F. (1950). Dimensions of similarity. American Journal of Psychology, 3, 516-556.

BAIRD, J. C., \& Noma, E. (1978). Foundations of scaling and psychophysics. New York: Wiley.

Beals, R., Krantz, D. H., \& TVersky, A. (1968). Foundations of multidimensional scaling. Psychological Review, 75, 127-143. 
Birnbaum, M. H. (1982). Controversies in pscyhological measurement. In B. Wegner (Ed.), Social attitudes and psychological measurement. Hillsdale, NJ: Erlbaum.

Borg, I., \& LEUTNER, D. (1983). Dimensional models for the perception of rectangles. Perception \& Psychophysics, 34, 257-267.

Carroll, J. D., \& Chang, J. J. (1970). Analysis of individual differences in multidimensional scaling via an $\mathrm{N}$-way generalization of Eckart-Young decomposition. Psychometrika, 35, 283-320.

EIsLER, H. \& RosKam, E. E. (1977). Multidimensional similarity: An experimental and theoretical comparison of vector, distance and set theoretic models. Acta Psychologica, 5, 1-46, 335-363.

EKMAN, G. (1963). A direct method for multidimensional ratio scaling. Psychometrika, 28, 33-41.

ENGEN, T., \& LeVY, N. (1955). The influence of standards on psychophysical judgments. Perceptual \& Motor Skills, 5, 193-197.

GigerenZER, G. (1983). Über die Anwendung der Informationsintegrationstheorie auf entwicklungspsychologische Problemstellungen: eine Kritik. Zeitschrift fü Entwicklungspsychologie und Pädagogische Psychologie, 15, 101-120.

GutTman, L. (1977). What is not what in statistics. The Statistician, 26, 81-107.

Horan, C. B. (1969). Multidimensional scaling: Combining observations when individuals have different perceptual structures. Psychometrika, 34, 129-165.

HYMAN, R., \& WELL, A. (1967). Judgments of similarity and spatial models. Perception \& Psychophysics, 2 , 233-248.

KRANTZ, D., \& TVERSKY, A. (1975). Similarity of rectangles: An analysis of subjective dimensions. Journal of Mathematical Psychology, 12, 4-34.

Micko, H. C., \& Fischer, W. (1970). The metric of multidimensional psychological spaces as a function of the differential attention to subjective attributes. Journal of Mathematical Psychology, 11, 118-143.

NomA, E., \& JoHnson, J. (1977). Constraining nonmetric multidimensional scaling configurations (Tech. Rep. No. 60). Ann Arbor: University of Michigan, Human Performance Center.

Parduccl, A. (1982). Category ratings: Still more contextual effects. In B. Wegner (Ed.), Social attitudes and psychophysical measurement. Hillsdale, NJ: Erlbaum.

Plateau, J. A. F. (1872). Sur la mesure des sensations physiques, et sur la loi qui lié l'intensité et de sensations a l'intensite de la cause excitante. Bulletins de l'Academie Royale des Sciences, des Lettres et des Beaux-Arts des Belgique, 33, 376-385.

Poulton, E. C. (1968). The new psychophysics: Six models for magnitude estimation. Psychological Bulletin, 69, 1-19.

Restle, F., (1961). Psychology of judgement and choice. New York: Wiley.

RosКАм, E. E. (1972). An algorithm for multidimensional scaling by metric transformations of data [Program Bulletin No. 23 (106)]. Holland: Universiteit Nijmegen.

SchönemanN, P. H. (1972). An algebraic solution for a class of subjective metrics models. Psychometrika, 37, 441-451.

SCHÖNEMANN, P. H. (1977). Similarity of rectangles. Journal of Mathematical Psychology, 16, 161-165.

SCHönemanN, P. H. (1979). On possible psychophysical maps: I. Quadratic transformations. Bulletin of the Psychonomic Society, 14, 329-332.

SchönemanN, P. H. (1980). On possible psychophysical maps: II. Projective transformations. Bulletin of the Psychonomic Society, 15, 65-68.

Schönemann, P. H. (1982). A metric for bounded response scales. Bulletin of the Psychonomic Society, 19, 317-319.

SCHÖNEMANN, P. H. (1983). Some theory and results for metrics for bounded response scales. Journal of Mathematical Psychology, 27 311-324.

SCHÖnEMANN, P. H., \& Borg, I. (1981). On the interaction between area and shape. In I. Borg (Ed.), Multidimensional data representations: When and why. Ann Arbor: Mathesis.

SchönemanN, P. H., James, W. L., \& Carter, F. S. (1978). COSPA, common space analysis-a program for fitting and testing Horan's subjective metrics model. Journal of Marketing Research, 12, 287-290.

SchönemanN, P. H., KienapPle, K. (1984). Artefakte in der mehr- dimensionalen Skalierung. Zeitschrift für Experimentelle und Angewandte Psychologie, 31, 483-506.

Schulz, U. (1971). Über zwei Modelle der multidimensionalen Skalierung unter Berucksichtigung individueller Differenzen. Unpublished doctoral dissertation, Philipps-Universität, Marburg, West Germany.

SchULZ, U. (1975). Zu einem Dekompositionsmodell der multidimensionalen Skalierung mit individueller Gewichtung der Dimensionen. Psychologische Beitrage, 20, 219-246.

ShEPARD, R. N. (1962). Analysis of proximities: Multidimensional scaling with an unknown distance function. Psychometrika, 27, 125$140,219-246$.

SixtL, F., \& WENDER, K. (1964). Der Zusammenhang zwischen multidimensionalen Skalieren und Faktorenanalyse. Biometrische Zeitschrift, 6, 251-261.

TAKANE, Y. (1981). Multidimensional successive category scaling: A maximum likelihood method. Psychometrika, 46, 9-28.

Teghtsoonian, M. \& Teghtsoonian, R. (1971). How replicable are Stevens's power law exponents for individual subjects? Perception \& Psychophysics, 10, 147-149.

Teghtsoonian, R. (1971). On the exponents in Stevens' law and the constant in Ekman's law. Psychological Review, 78, 71-80.

TVersky, A. (1977). Features of similarity. Psychological Review, 84, 327-352.

TVersky, A., \& GATI, I. (1982). Similarity, separability, and the triangle inequality. Psychological Review, 89, 123-154.

WENDER, K. (1969). Die psychologische Interpretation nichteuklidischer Metriken in der multidimensionalen Skalierung. Unpublished doctoral dissertation, Technische Hochschule Darmstadt, West Germany.

WENDER, K. (1971). A test of independence of dimensions in multidimensional scaling. Perception \& Psychophysics, 10, 30-32.

Wiener-EhrLiCH, W. K. (1978). Dimensional and metric structures in multidimensional stimuli. Perception \& Psychophysics, 24, 399-414.

\section{NOTES}

1. Such stimulus material is well suited to a study of the orientation question, that is, in the present case, whether subjects utilize a subjective coordinate system parallel to height and width $(\mathrm{H} / \mathrm{W})$ or a system parallel to area and shape (A/S), which are the two obvious choices at the subject's disposal. Since the design coordinates were logged physical coordinates, a rotation of the (logged) H/W system through $45^{\circ}$ carries it into a (logged) $\mathrm{A} / \mathrm{S}$ system.

2. Alternatively subjects might first add the line segments and then contract this sum. It is clear that the MBR in the form $(a+b) /(1+a b)$ also admits this alternative interpretation. Another simple metric that achieves this is $\mathrm{d}$ : $=a+b-a b=1-(1-a)(1-b)$, with $0 \leq a, b \leq 1$. Its predictions do not depart substantially from those of the MBR (see Schönemann, 1983, p. 316). Although its standard sequence is simpler, it is more difficult to invert than the MBR (Equations 6 and 7).

3. The range reduction in MBR(sL) and MBR(sMX) introduces two minor computational complications: (1) Since the inverse hyperbolic tangent map exists only over the half-open interval $[0,1)$, all dissimilarities exceeding the reduced bound have to be scaled back before this transformation can be applied to analyze the transformed data in the CB mode. (2) Since the MRSCAL least squares solution is computed on relative dissimilarities normed with respect to $\mathrm{sL}(<\mathrm{L})$, these relative distances will be larger than those relative to $L$ used to evaluate the fit for $C B$ and $\operatorname{MBR}(\mathrm{L})$. Hence, the coordinate matrix returned by the program will be too large, so that the residuals $g(a, b)-c$ will also be too large. This bias can be removed by computing a fractional scalar multiple of the coordinate matrix returned by the program, to retain strict comparability for the residuals $e=g(a, b)-(d * / 9)$. This uniform reduction in size does not affect the shape of the stimulus configuration obtained for MBR(sL).

Perhaps a simpler alternative would have been to compute the residuals for the MBR(sL) as $g(a, b)-d^{*} / 7.2$, and then to multiply them by $7.2 / 9$, to restore comparability with those left by $\mathrm{CB}$ and $\mathrm{MBR}(9)$.

(Manuscript received October 15, 1984; revision accepted for publication June 11,1985 .) 